
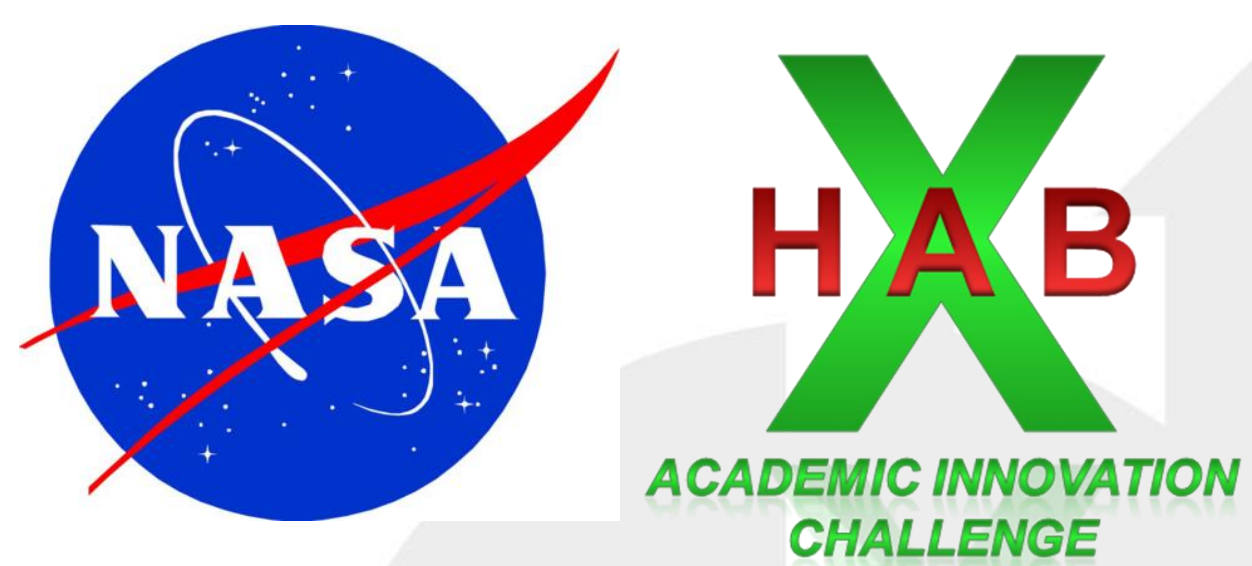

\title{
A Novel Approach for Engaging Academia in Collaborative Projects with NASA through the $\mathrm{X}$-Hab Academic Innovation Challenge
}

\section{Tracy R. Gill}

\section{NASA/Kennedy Space Center}

AIAA Space 2015, Aug 31 - Sep 2, 2015

Pasadena, California 


\section{Outline}

- Introduction

- Process and Timeline

- History

- Benefits

- Future Plans

- Conclusion

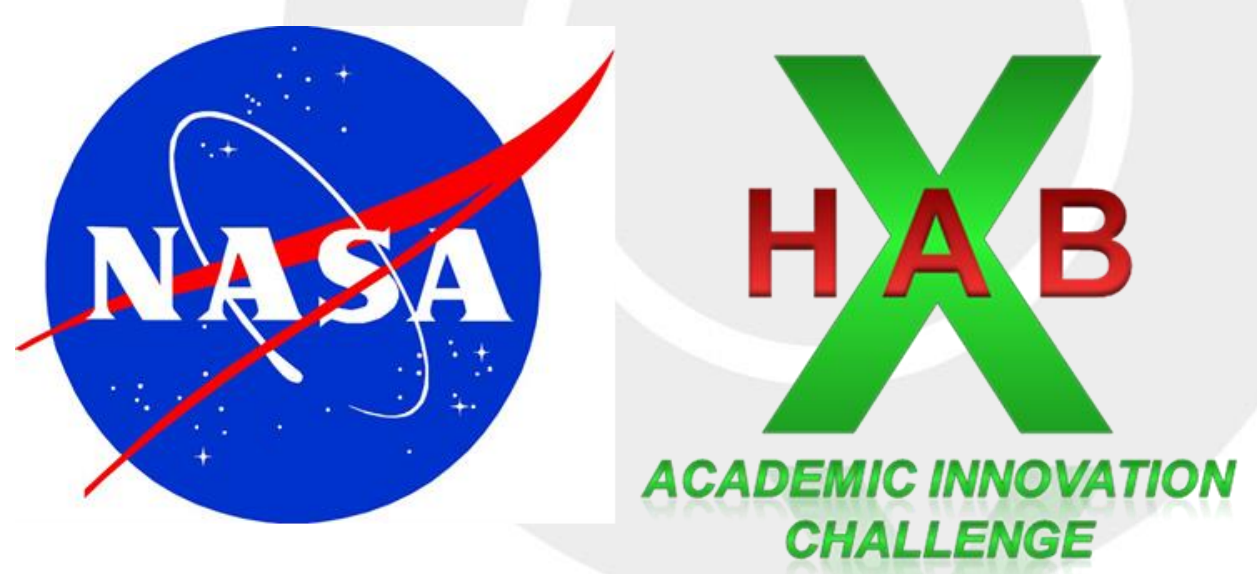




\section{X-Hab Introduction}

- X-Hab Academic Innovation Challenge is currently in its Sixth Year of Execution

- It is run through KSC for NASA Human Exploration Operations Mission Directorate

- Solicitation partnering NASA and academia on technologies and studies for deep space missions

- NASA awards grant funds ( $\$ 10 \mathrm{k}-\$ 50 \mathrm{k})$ to design and produce functional products of interest

- Universities propose on a variety of projects suggested by NASA and are then judged on technical merit, academic integration, leveraged funding, and outreach. 


\section{X-Hab Introduction (cont'd)}

- Universities assemble a multi-discipline team of students and advisors that invest months working together, developing concepts, and frequently producing working prototypes.

- Students gain a quality experience, working real world problems that have the possibility to be implemented, and they work closely with subject matter experts from NASA who guide them through a formal engineering development process

- The first X-Hab in 2011 was run as a head to head competition. After that, it was decided to pursue a diverse portfolio of projects each year to gain more from the investment 


\section{Notional Selection Schedule}

- February Solicit project ideas, funding for projects in next fiscal year

- Early March Release solicitation

- Early April Questions for online technical interchange due

- Mid April Responses for questions published online

- Late April Proposal due date

- Late May Award announcements and announce selections for the fall

- June

Collect project funding and obligate to NSGF 


\section{Project Execution Schedule}

- September Project kickoff meetings for new projects

- October Requirements and System Definition Review

- November Preliminary Design Review

- January Critical Design Review

- March Progress Checkpoint Review

- May Project Completion and Evaluation by NASA 


\section{OAIAA}

\section{X-Hab 2011}

\section{- Inflatable Habitat Loft competition}

- University of Maryland (bottom right), Oklahoma State University (right), and University of Wisconsin (below)
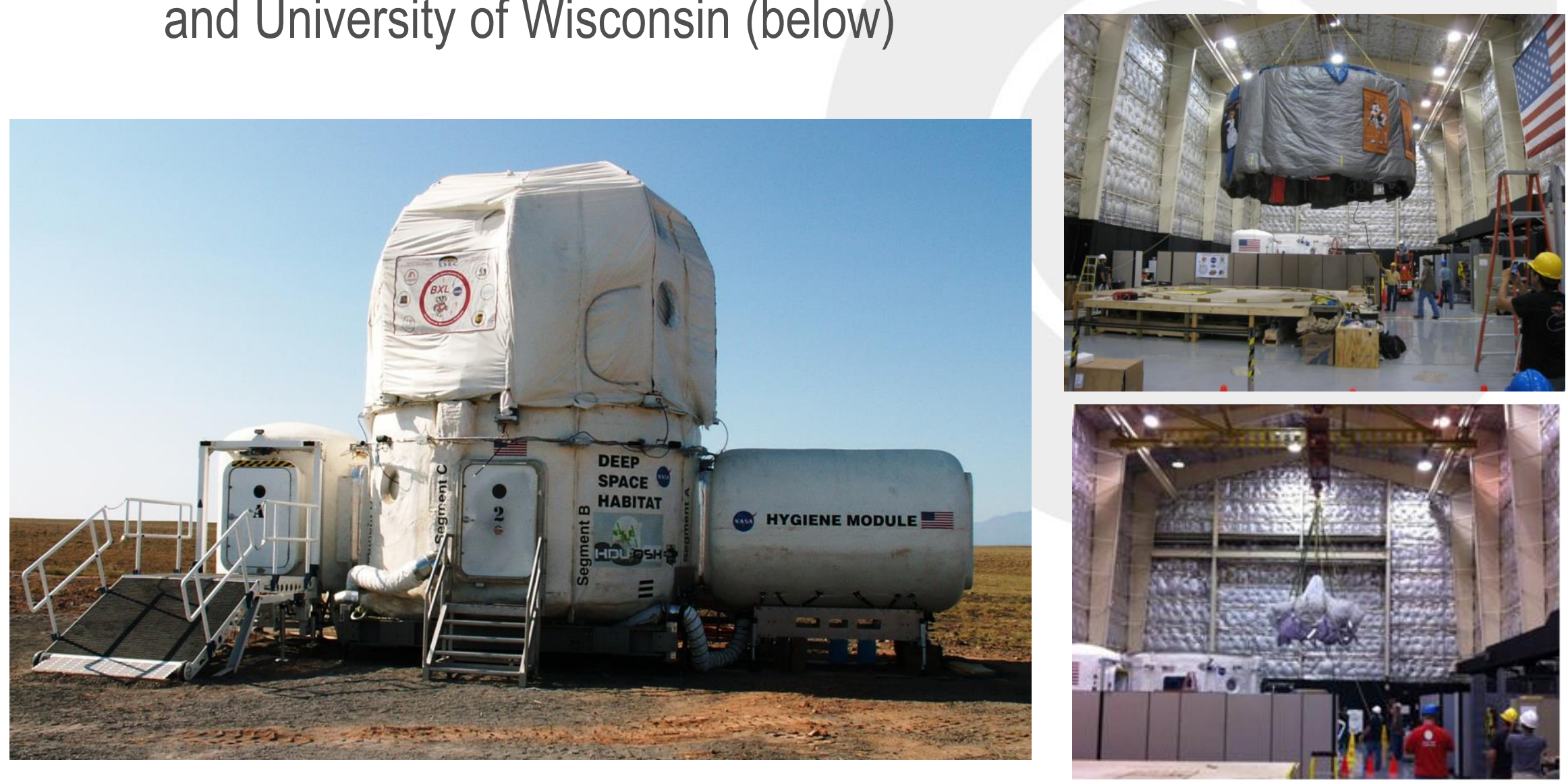
X-Hab 2012

- Ohio State University: Plant Growth Monitoring System

- University of Bridgeport, Connecticut: Sample Handling System for GeoLab glovebox (pictured)

- University of Maryland, College Park: vertically-oriented habitat study.

- Oklahoma State University: horizontally-oriented habitat study.
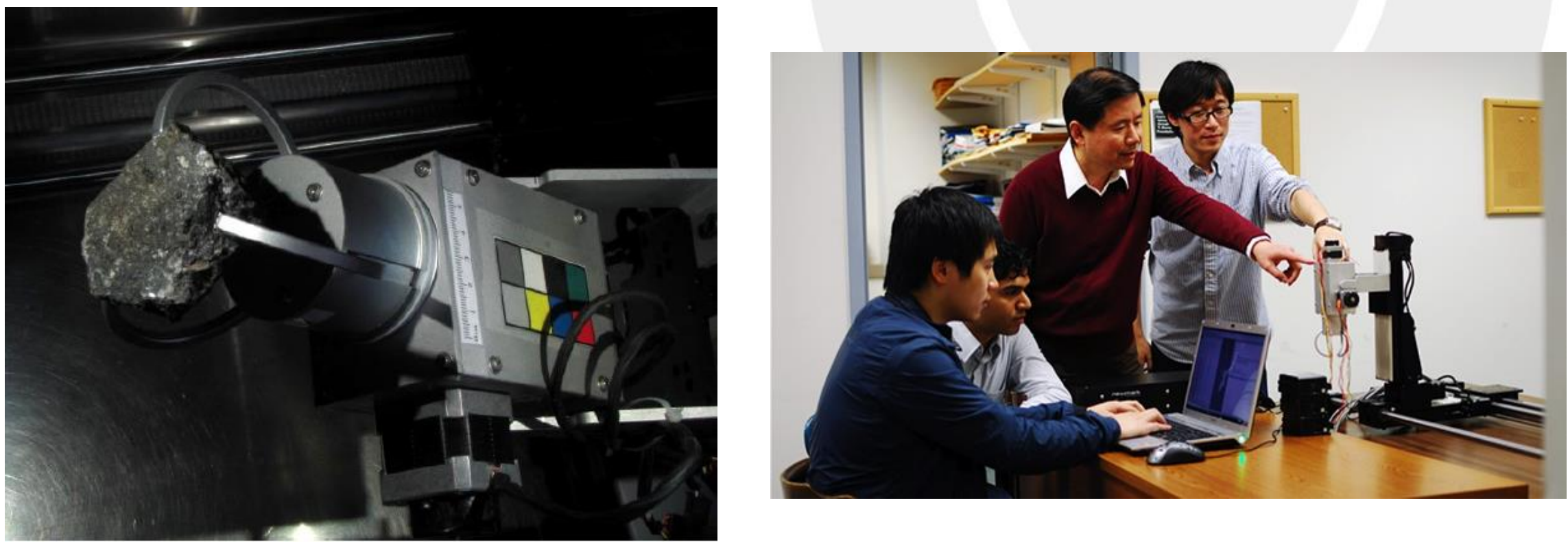


\section{X-Hab 2013}

- University of Alabama, Huntsville: Microgravity Random Access Stowage \& Rack System

- California Polytechnic State University: Vertical Habitability Layout and Fabrication Studies (pictured)

- Oklahoma State University: Horizontally Oriented Inflatable Deep Space Habitat

- Texas A\&M University: Wireless Smart Plug

- University of Colorado: Remote Plant Food Production Capability

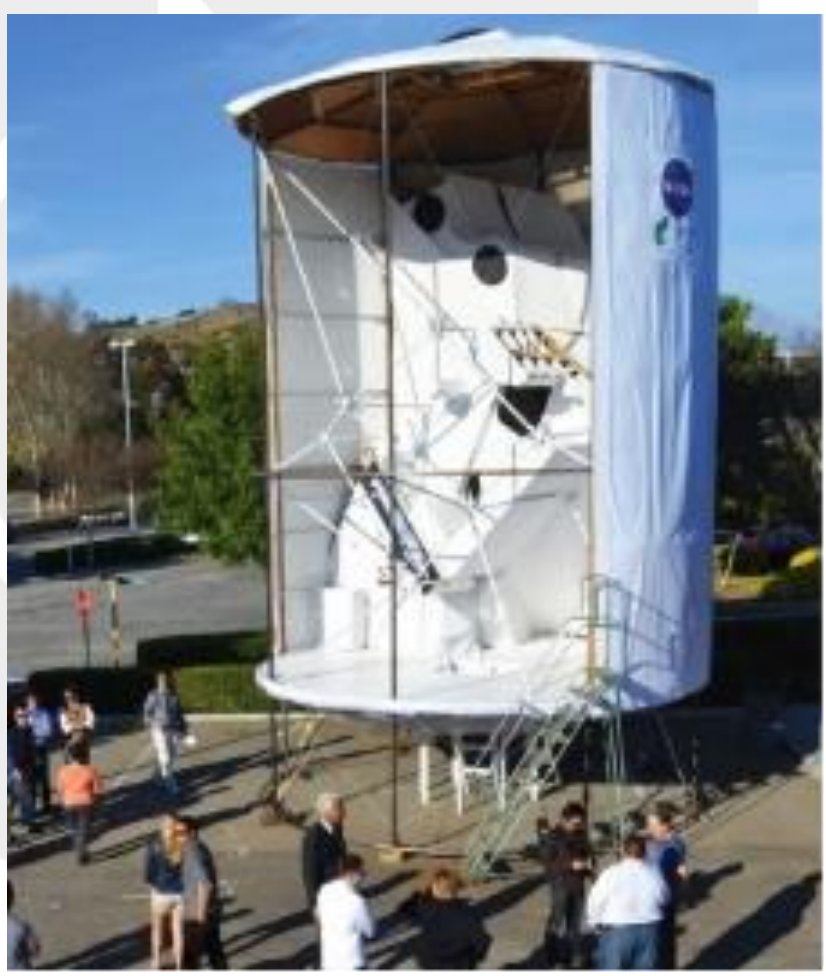




\section{X-Hab 2014}

- University of Wisconsin, Madison: Badger Compartmentalized Onboard Material Extrusion Technology

- University of South Alabama, Mobile: Closed Environment Air Revitalization System Based on Metal Organic Framework Adsorbents

- Rice University, Houston: SpaceRing: a Versatile, Scalable Power-Generation and Cooling System

- University of Colorado at Boulder: Plant Anywhere: Plants Growing in Free Habitat Spaces (pictured)

- University of Maryland, College Park (2 projects): Vertical Habitability Layout Studies, Neutral Buoyancy/Parabolic Flight Habitat Studies

- Oklahoma State University, Stillwater: Horizontal Habitability Layout Studies
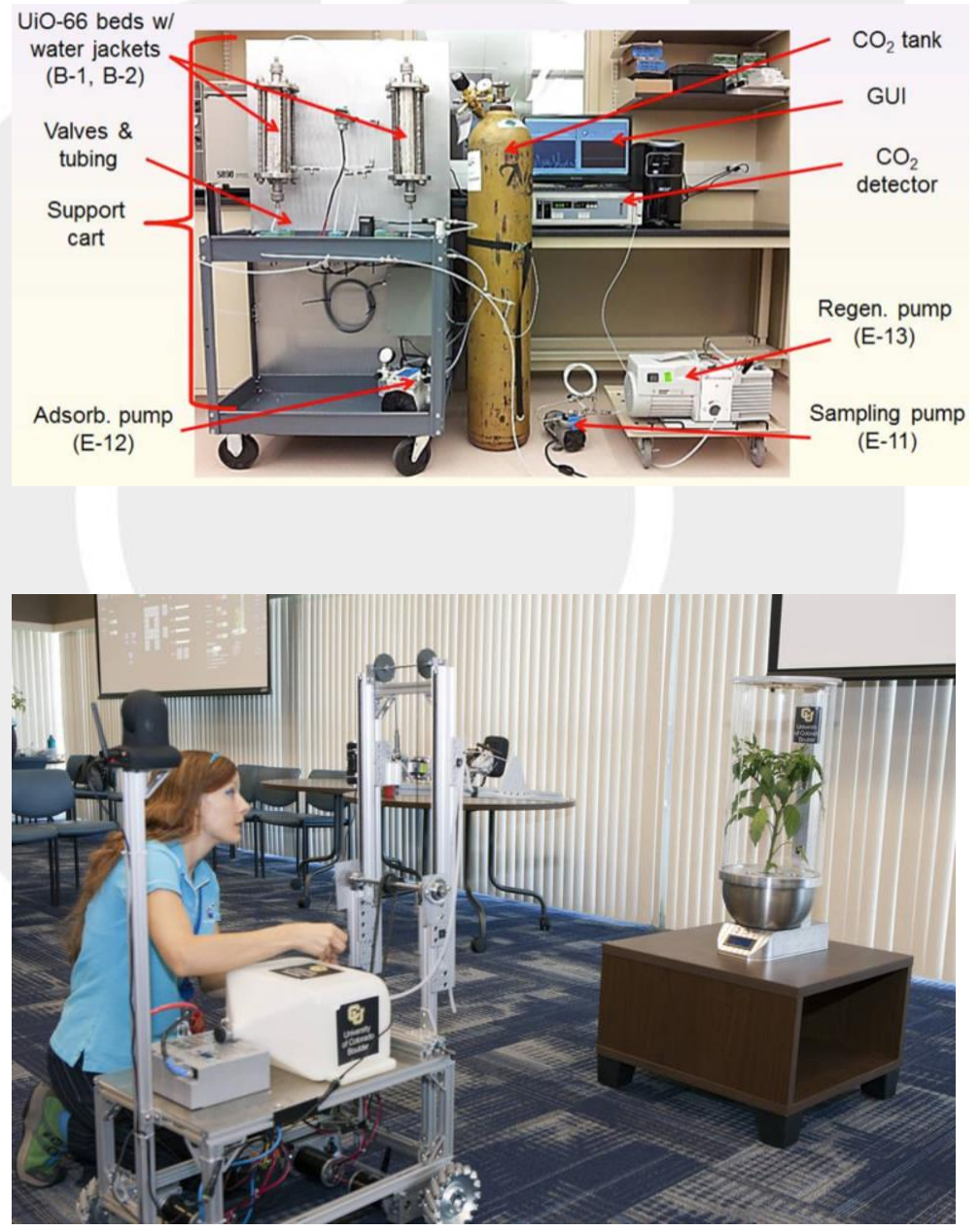


\section{X-Hab 2015}

- University of Colorado at Boulder: Deployable Greenhouse for Food Production

- Oklahoma State University: Deployable Greenhouse for food production on longduration exploration missions

- University of South Alabama: Development of a Volumetric Absorption System for $\mathrm{CO} 2$ and $\mathrm{H} 2 \mathrm{O}$ Multicomponent Isotherm Measurements

- University of Vermont: Design of a "SmartStructure" Deployable Airlock (top)

- University of Wisconsin - Milwaukee: Design of a Carbon-fiber/Fused Deposition Modeling Spacecraft Structural Fabrication System (bottom)
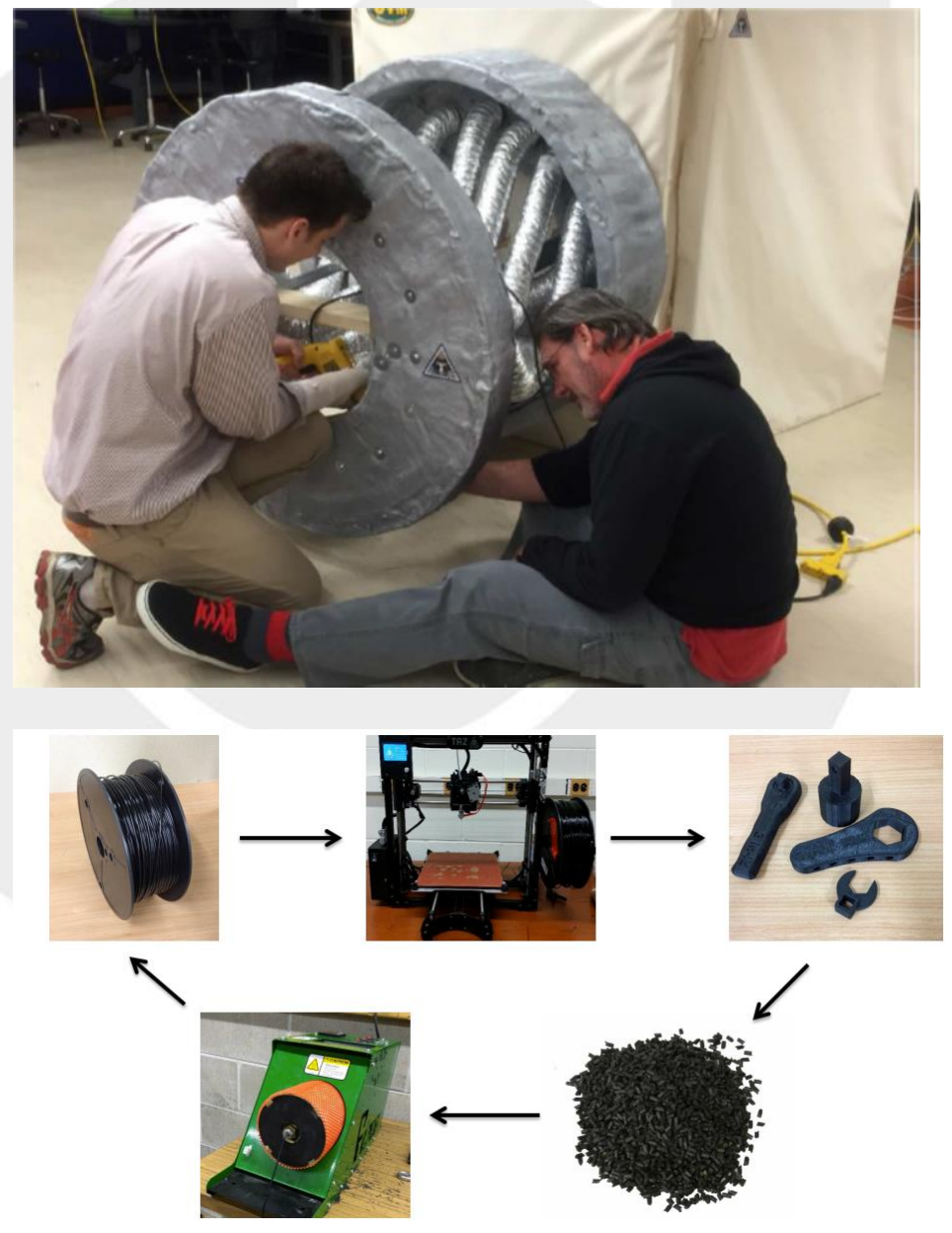


\section{X-Hab 2016 (Sept 2015 kickoffs)}

- University of Maryland, College Park, Md. - Inflatable/Deployable Airlock Structures

- Pratt Institute, Brooklyn, N.Y. - Human Centered - Designs for Mars Transit Habitat

- Oklahoma State University, Stillwater, Oklahoma - Deep Space Mars Transit Habitat Layout Studies

- University of South Alabama, Mobile, Alabama - Development of a Concentration Swing Frequency Response Device

- University of Puerto Rico at Mayagüez, Puerto Rico - Technology Development of Low-Power Required Manufacturing of Metals for the Zero Gravity Environment Project

- Utah State University, Logan, Utah - Student Experimental Microgravity Plant System (SEMPS)

- The Ohio State University, Columbus, Ohio - Water Assurance: Improve water delivery of a modular vegetable production system

- University of Colorado-Boulder, Boulder, Colo. - Performance Characterization and Enhancement of the MarsOASIS Space Plant Growth System 


\section{X-Hab Selections 2011-2016}

\section{X-Hab 2015}

University of Wisconsin - Milwaukee

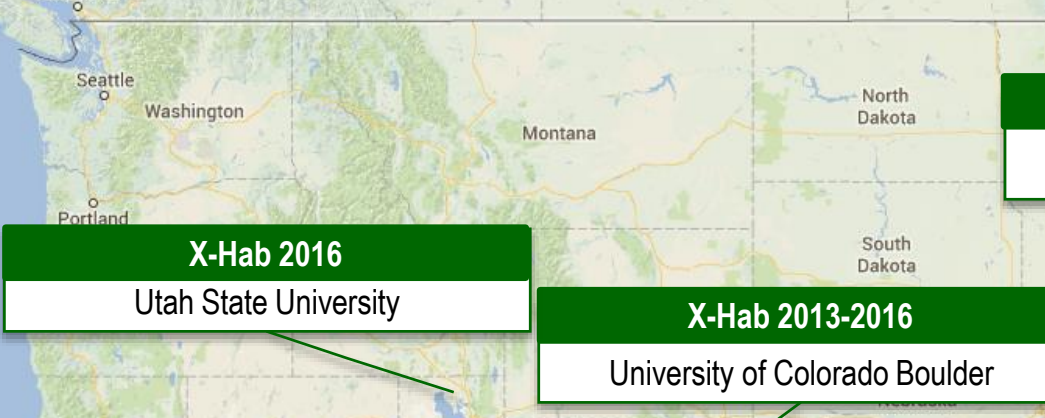

X-Hab 2013

California Polytechnic University
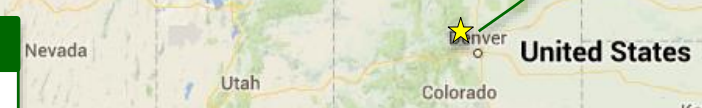

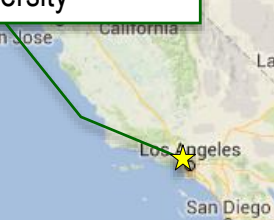

\section{X-Hab 2011-2016} Oklahoma State University

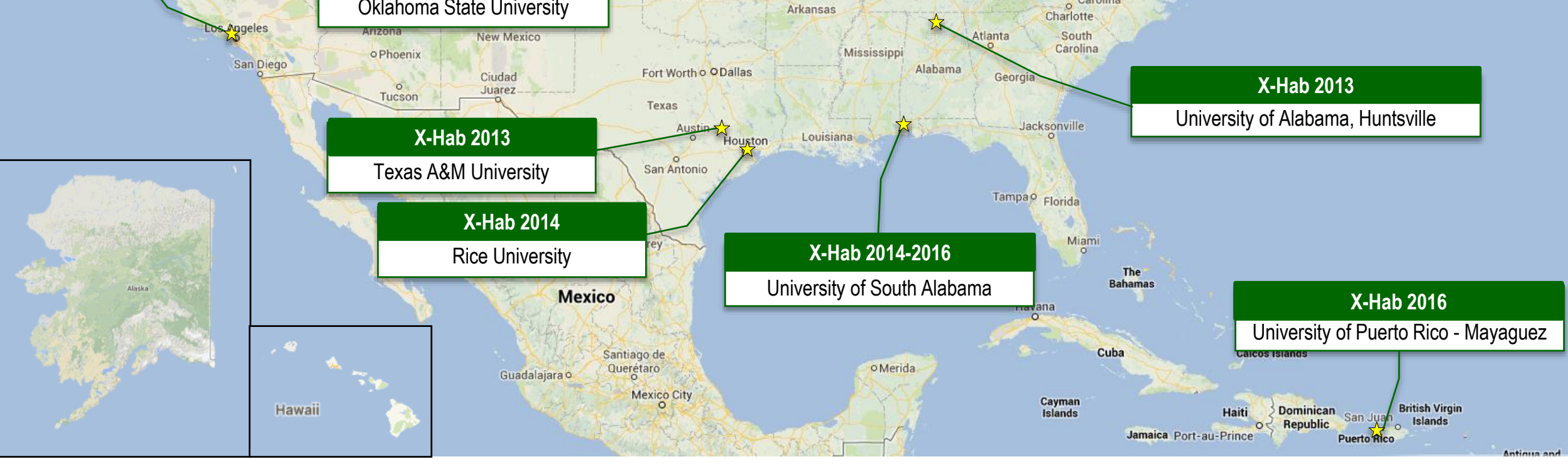

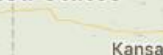

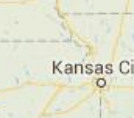

New Mexico

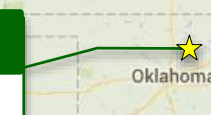

Xab 2013

\section{X-Hab 2011, 2014}

University of Wisconsin - Madison

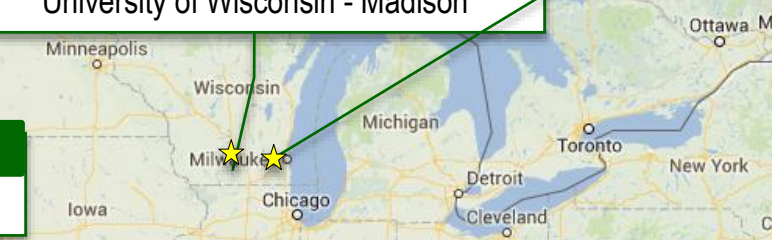

Minneapols lowa

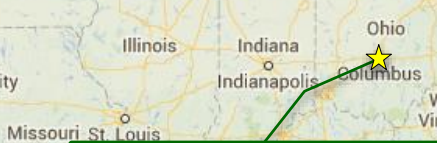

$$
\text { X-Hab } 2016
$$

Ohio State University

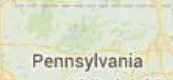

Philadelphia

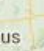

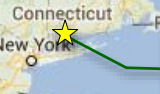

connech

Marylarz

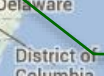

X-H

University of Bridgeport

X-Hab 2016

University of Vermont

University of Maryland 


\section{Benefits}

- Reports and Prototypes

- NASA gets products in which they are interested at low cost

- Outreach

- Teams do excellent outreach nationally and locally in conferences, schools, and various media

- Financial Flexibility

- Cooperative Agreement with National Space Grant Foundation facilitates solicitation ahead of academic year

- Student Experience

- Good project cycle experience ahead of employment 
Future Plans

- X-Hab has been run under one project within HEOMD's Advanced Exploration Systems Division

- Now supports multiple divisions in HEOMD and projects within AES

- Collaboration has become the norm rather than the exception with limited funds in architecture and technology development activities

- X-Hab can continue to grow to support HEOMD activities and engage the creativity of academia 


\section{Conclusion}

- The X-Hab Academic Innovation has evolved and matured as it enters its sixth year of operation.

- This model can serve as an excellent mechanism to engage academia in research and technology efforts not only within NASA but any government agency.

- The model benefits government with creative, low cost focused research through academic partners while the universities can tailor the curriculum to give their students a good systems engineering experience guided by the expertise of the agency. 


\section{Questions?}

- Thanks to the following folks:

- Kriss Kennedy at JSC who created the concept of X$\mathrm{Hab}$

- All the civil servants and contractors around NASA who have supported X-Hab project teams

- National Space Grant Foundation

- The university teams and their partners who have been so successful in executing projects. 


\section{For more information}

- NASA X-Hab Academic Innovation Challenge Public Page

- http://www.nasa.gov/exploration/technology/deep space habitat/xhab

- NASA Deep Space Habitat Facebook page

- https://www.facebook.com/pages/NASA-Deep-SpaceHabitat/303267556447857

- National Space Grant Foundation (NSGF) X-Hab site

- https://www.spacegrant.org/xhab

- Tracy R. Gill

- NASA/Kennedy Space Center

- Tracy.R.Gill@nasa.gov 


\section{OJIAa}

Shaping the Future of Aerospace 